\title{
Energy Efficiency with Computational Topology and Routing in Wireless Sensors Networks
}

\author{
Fahad Mohammed Bahazaq and Vijey Thayananthan \\ Computer Science Department, \\ Faculty of Computing and Information Technology, \\ King Abdul Aziz University, \\ Jeddah 21589, Saudi Arabia.
}

\begin{abstract}
Computational topology and routing are the major area where energy is needed to activate a number of functions integrated in wireless sensor network. In this research, reducing energy consumption and increasing energy efficiency (EE) are the main aims when specific algorithm of routing protocol and topology are employed in WSN. In order to solve the main points through this research, we studied the novelties of the routing protocols based on ant colony optimization (ACO). In these studies, accurate distance between the sensor nodes based on the specific topology is also analyzed. From these approaches EE can be enhanced in future WSNs which have either 2D or 3D topologies.
\end{abstract}

\section{General Terms}

Computational topology (CT) and routing are considered as my general terms. Throughout this research, EE analysis is considered with $\mathrm{CT}$ and routing.

\section{Keywords}

Energy aware algorithms, EE, topologies, CT, routing protocols, ACO, WSN

\section{INTRODUCTION}

Energy analysis of WSNs is a popular topic in current wireless technology. Topologies are varied with applications such as environmental monitoring, under water protections etc. Improvements in the EE with $\mathrm{CT}$ should be analyzed because wireless devices between the source and destination are still consuming high power. WSNs usually contain thousands of network devices that hold a lot of sensors, which are randomly and widely deployed [3]. Once deployed, these sensor nodes are usually inaccessible to the user, thus replacement of the energy resource is not good option. Therefore, EE in the wireless network is the main design issue that needs to be considered with routing protocols which help to improve the life time and power consumption of the entire network.

Regarding the energy algorithms, investigations and analysis, the design of general or specific media access control (MAC) and routing protocols for WSNs has been considered in a number of publications. However, only a few have been concerned with design parameter such as energy optimization of packet size, congestions, link quality, delivery time etc. Single hop known as point to point communication topology is mentioned in many applications which need reasonable energy [15][17]. But maximizing the throughput with packet size optimization in this topology is also addressed when EE is improved in WSN using enhanced routing protocols.

Topologies and CT are key terms for maintaining and analyzing WSNs in real applications. Here, topologies of 2D and 3D WSNs and considered for EE analysis, which depends on the inference capability of routing topology designed for routing improvement, CT, effective WSN management etc [19]. In the CT approach, novel geometrical methods are considered to solve the various architectures of the WSN applications. Both topology control and routing problems are used for enhancing EE, guaranteeing the packet delivery and optimizing the distances.

Routing protocols need special attention when they are designed for specific WSN applications they are such as simplicity, EE and scalability [3]. EE can be enhanced without increasing complexity which depends on the optimization of routing protocols and optimum design of WSN. Increasing complexity and CT needs more power, hence nodes which are basically routers in WSN behavior and properties should be considered with routing protocols that minimize the energy consumption and CT. There are many type routers and routing protocols used in which WSN is being activated with different topology designed for fixed and mobile applications. In this research, ACO is modified with a combination of energy, congestion and link quality.

In the research field of WSNs, reduction in energy consumption of CT so that the lifetime of WSNs can be prolonged is one of the hottest spots. A salient feature of WSN powered by battery or solar is its extremely constrained source of energy supplied by batteries coming with sensor nodes, because sensor nodes are typically small and thus use tiny batteries [9][13]. The lifetime of WSN is defined as the time for the first node or a certain percentage of network nodes to run out of power [1][16]. The basic operation in sensor network is the systematic gathering of sensory data to be eventually delivered to the base station or sink node. Since the sensor nodes consume a lot of energy, communication between the base station and the sensors must be carried out in an energy-efficient manner [14].

The remainder of the research paper is organized as follows. we have briefly described the related works of energy efficient concepts based on routing and CT in Section 2. Proposed protocol is explained in Section 3. In addition to this, definition and modifications of ACO also considered. In Section 4, flowchart of the algorithm and necessary equations for energy consumption in both transmitter and receiver are explained. Performance results and analysis are described in Section 5 for the EE improvement in selected WSN, where we vary the network size, topology, and the deployment area. Section 6 concludes the paper.

\section{RELATED WORKS}

There are many publications delivered to develop EE algorithms and protocols in WSNs which are applicable in specific and multi mode developments [5][7]. Here, routing concepts based on CT, EE algorithms, etc, are briefly outlined 
for our research which looks for some enhancement in EE through the design of routing protocols.

Basically, there are three categories of routing protocols proposed in previous articles they are proactive, reactive, and the combination of these two [18].

\subsection{Energy consumption}

Minimum energy consumption with low-cost technology is the ultimate target in all cases of WSN. In real applications, nodes of the WSN are in 3D architecture which has active and non-active sensors located in nodes. As given in (1), distance between the sensor nodes should be calculated accurately according to $3 \mathrm{D}$ and $\mathrm{CT}$ concepts.

$d=f(x, y, z)$

Here, $\mathrm{x}, \mathrm{y}$ and $\mathrm{z}$ are the co-ordinates of $\mathrm{x}-\mathrm{y}$ points on horizontal planes and vertical point respectively.

\subsection{Computational topology}

There are many topologies employed in different applications of WSN. In all WSN architecture, CT is analyzed for many reasons they are such as reducing the complexity, increasing data rate, optimizing $\mathrm{EE}$, etc. In this research, we have analyzed the EE through the $\mathrm{CT}$ and routing strategies, which depend on the energy-efficient routing protocols. In order to explain about CT, figure 1 can be illustrated for 3D WSN.

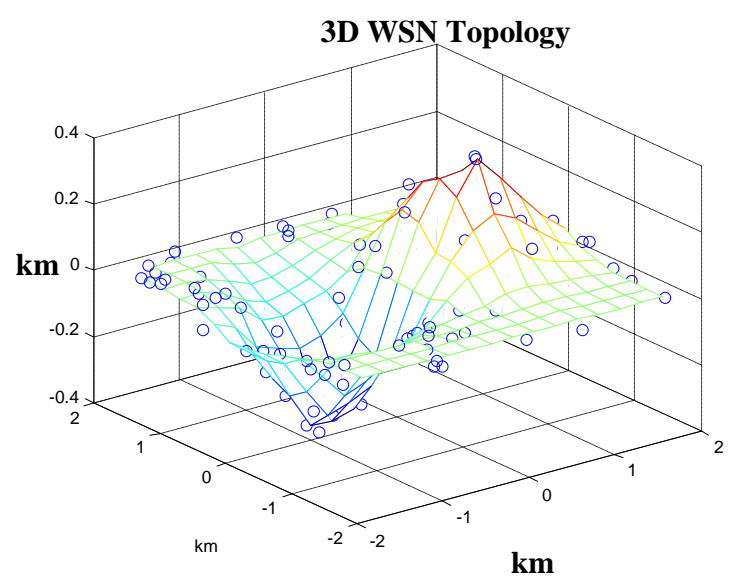

Fig 1: Topology and routing design for 3D WSN

Further, necessary calculations of CT are obtained from the number of sensor nodes located in the architecture of WSN. As shown in figure 1, sensor nodes are deployed within three dimensional invisible box which covers 16 square $\mathrm{km}$ area and $0.8 \mathrm{~km}$ high or deep. As shown in the table1, following analysis can be used for future developments

Table 1. Comparisons of WSN architectures

\begin{tabular}{|c|c|c|c|}
\hline Architecture & EE & Complexity & CT \\
\hline 2D & $90 \%$ accurate & $\mathrm{O}\left(\mathrm{N}^{2}\right)$ & low \\
\hline 3D & $98 \%$ accurate & $\mathrm{O}\left(\mathrm{N}^{3}\right)$ & high \\
\hline
\end{tabular}

Simple example as shown in figure 1 is used for comparisons of two architectures and $\mathrm{N}$ is the size of array used in them.

\subsection{Routing strategy}

In this section, EE is considered with routing concepts which mean that the design of the routing, software, firmware and hardware architectures and optimization of routing complexity are very important points applied in the CT and development processing. If a router is designed with above mentioned points, EE can be achieved but commercial products of current router expect many features which take a lot of power. So, EE can be achieved not only the efficient routing concept and CT but also it depends on the customers' demand that they need more features. Even if efficient routing protocols are employed in the WSNs, more features need more power.

\subsubsection{Energy-efficient routing protocols}

As energy-efficient schemes, Equalized Cluster Head Election Routing Protocol (ECHERP), Energy Efficient and Load Balancing Routing can be used in WSNs. Ring-based Energy Aware Routing (REAR) protocol can also be used in WSNs because it can achieve both energy balancing and EE for all sensor nodes through CT. Ad-hoc On-demand Distance Vector Routing (AODV) which is a reactive routing protocol, Destination-Sequenced Distance Vector (DSDV) routing which is a proactive [18] routing protocol and Low-Energy Adaptive Clustering Hierarchy (LEACH). The above mentioned algorithms and their CT concepts are different because same topology is approached with different routing protocols. Geographic Routing with Environmental Energy Supply (GREES) is an algorithm that makes routing decisions combining geographic and energy efficient through CT and routing techniques.

\subsubsection{Energy saving with ACO}

The optimized routing protocols depend on the $\mathrm{CT}$ which allows us to construct the energy saving protocols based on ACO they are such as EEABR (Energy-efficient ant based routing), ACORC (ant colony optimization router chip), etc. The ACO concept for getting the shortest path [4] between the source and destination can be improved through efficient CT techniques. ACO algorithm is a technique for solving the computational problems which can be reduced by finding good paths thorough graphs. There are various applications where ACO is used like finding the optimal path in Travelling Salesman Problem, Network Model Problem, Quadratic Assignment Problem and Vehicle Routing Problem [10][11].

\section{PROPOSED ROUTING PROTOCOL}

The proposed protocol based on ACO consists of 4 main steps. Firstly the user defines the number of nodes and the destination node in a WSN. Secondly the nodes in the network will be deployed as per the topology defined by the user. Thirdly the algorithm consists of various functions like nodes, final sender, final receiver, transmission, reception and path. Nodes tell us how many nodes have their radio "On". Final sender decides the nodes which act as final sender. Final receiver is decided based on the shortest path among active nodes. Except final sender and final receiver all other nodes radio are off to prevent energy wastage. After the final sender and final receiver decision have been made, data transmission takes place. Next is to check whether data is transmitted successfully or not and if not it would be transmitted for a specific number of times. The last step is to get the traversed path from source to destination (sink node).

\subsection{Definitions and assumptions}

In EE analysis, following definitions can be obtained with necessary assumptions.

EE is proportional to distance between the active nodes used in either $2 \mathrm{D}$ or $3 \mathrm{D} \mathrm{WSN}$. 
EE is proportional to distance between the base station and average location which is a mean distance of $2 \mathrm{D}$ and $3 \mathrm{D}$ WSN.

Assumption: Average distance of the sensor nodes is considered throughout of this research.

\subsection{Energy based ACO}

The energy (E) of the node depends on the power resource [12] such as battery, transmission during the route formation etc. In 3D WSNs, nodes are deployed in many directions but in order to calculate the energy between the two nodes, same (2) can be used. If more than two nodes are used closely, mean energy should be calculated.

$E=\left(E_{\max }-E_{\text {tot }}\right) / E_{\max }$

Where $E_{\text {tot }}$ and $E_{\max }$ are the total energy of processing and the maximum energy available to the node.

\subsection{Congestion based ACO}

Furthermore, because of ACO, ADHOP can handle problems in dynamic network topologies [6][8] to avoid congestion, broken routes, and improve the discovery and maintenance of routes. As in (3), congestion mainly depends on the delay ( $\left.D_{d e}\right)$, that affects the energy during the packet delivery.

$D_{\text {de }}=d_{\text {proc }}+d_{\text {prop }}+d_{\text {queue }}+d_{\text {trans }}$

Here $d_{\text {proc }}, d_{\text {prop }}, d_{\text {queue }}$, and $d_{\text {trans }}$ are processing, propagation queuing, and transmission respectively.

\subsection{Link based ACO}

The link quality ( $L$ ) which depends on the routing over best available path influenced with the quality of the radio channel affect the delivery of the packet during the transmission.

$L=\left(L_{\max -p}-L_{N}\right) / L_{\max -p}$

In (4), $L_{\max -p}$ and $L_{N}$ are maximum link power and noise power involved in the link.

\subsection{ACO based on ECL}

Our proposed protocols (ECL-ACO) can be described using above (section 3.2, 3.3 and 3.4) mentioned concepts. To design this protocol, routing metrics such as energy (E), congestion $(\mathrm{C})$ and link $(\mathrm{L})$ can be combined with ACO.

\section{DESIGN AND IMPLEMENTATIONS}

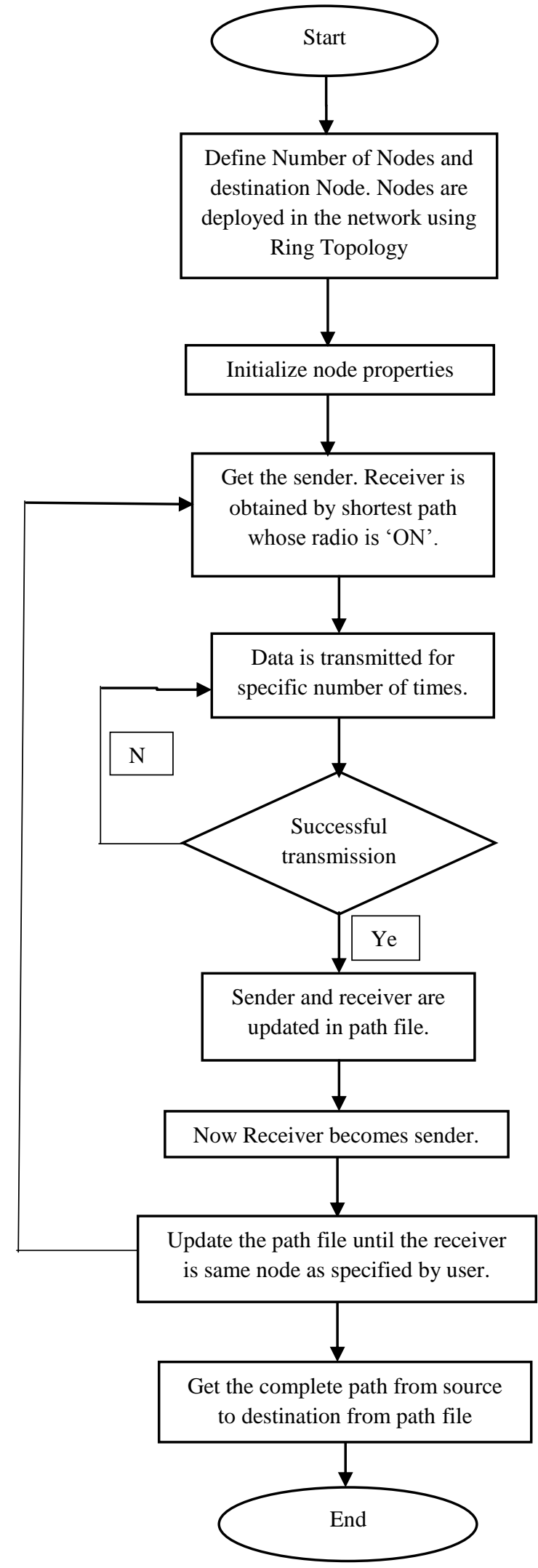

Fig 2: Flowchart of proposed research 
As shown in figure 2, flowchart of the modified ACO is designed. In ECL-ACO approach, same procedure can be used as in the flowchart. Following block diagram is representing the transmitting and receiving nodes of either $2 \mathrm{D}$ or 3D WSN. Although energy calculations depend on the individual node and link quality which influences with environmental conditions, ECL-ACO routing protocol plays an important role with distance $(\mathrm{PQ}=\mathrm{d})$ as shown in figure 3 .

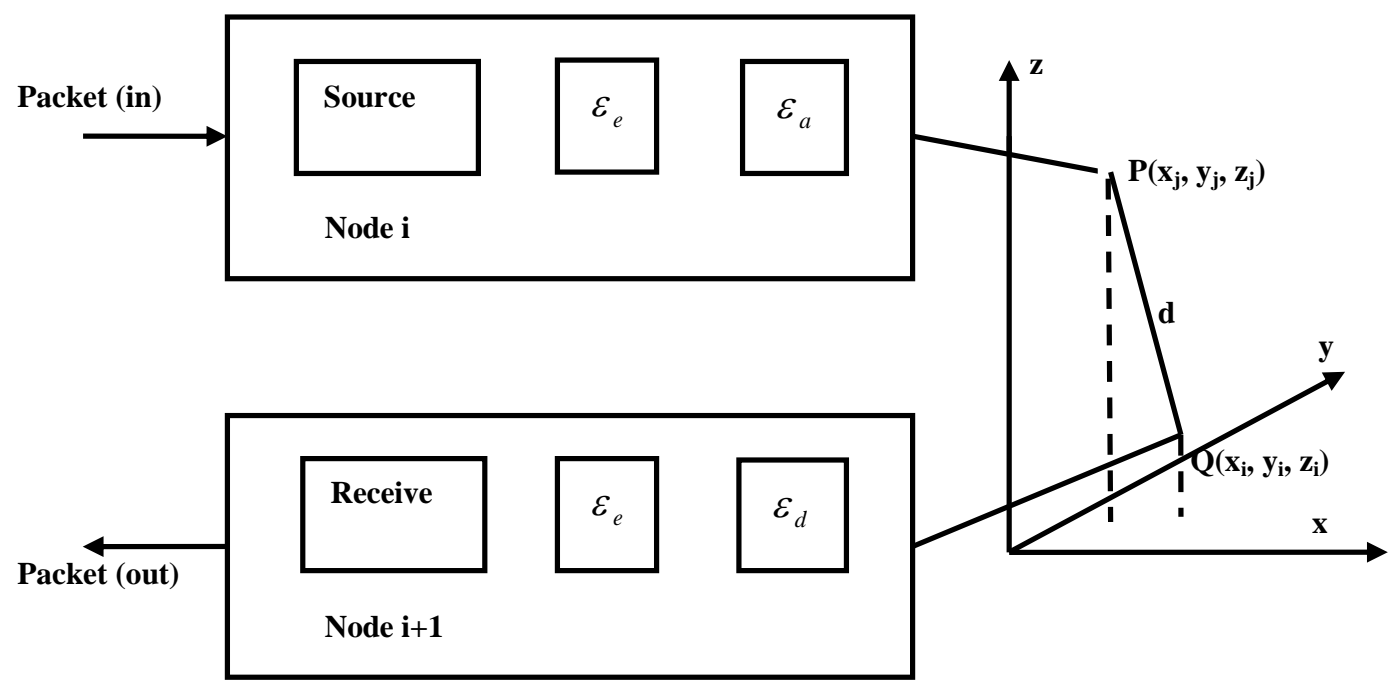

Fig 3: Necessary steps for total energy calculation

In figure 3, energy consumption is obtained from (5) and (6) when m-bit packet which holds the data between the two wireless sensor nodes. In following energy calculations, $d$ is the actual distance between the nodes.

$E_{t x}(m, d)=\varepsilon_{e} m+\varepsilon_{a} m$

Where $E_{t x}(m, d)$ is the transmitter energy consumption, and $E_{r x}(m, d)$ considered with electronic circuits and decoding is the receiver energy consumption given below.

$E_{r x}(m, d)=\varepsilon_{e} m+\varepsilon_{d} m$

The electronic circuits employed in both transmitter and receiver, consume energy $\mathcal{E}_{e}$ in each case. In the transmitter node, $\mathcal{E}_{a}$ is the circuits of amplifier energy which needs to be set with distance. To calculate the transmission energy in each node, following conditions (7) can be used in (5)

$\varepsilon_{a}=\left\{\begin{array}{l}\varepsilon_{b} \times d^{2}, \text { when } d<d_{0} \\ \varepsilon_{c} \times d^{4}, \text { when } d>d_{0}\end{array}\right.$

where $d_{0}$ is a threshold value. If the distance $\mathrm{d}$ is less than $d_{0}$, the free-space which is noise free propagation model can be applied. Otherwise, model is considered with multipath and fading channels which need more energy during the transmission [3]. Energy parameters $\varepsilon_{b}$ and $\varepsilon_{c}$ are influenced to adjust the amplifier energy with distance. According to the literature review [3], the $\varepsilon_{b}$ is set as $10 \mathrm{pJ} / \mathrm{bit} / \mathrm{m}^{2}$ and $\varepsilon_{c}$ is set as $0.0013 \mathrm{pJ} / \mathrm{bit} / \mathrm{m}^{4}$ in this research. When many nodes are allocated in the WSNs, average distance as in (8) is calculated to determine the total energy.
Average distance of nodes $d_{a}=d=\frac{\sum_{i}^{n} a_{i}}{n}$

In (8), $\mathrm{n}$ is the total number of nodes and is the $a_{i} \mathrm{i}^{\text {th }}$ node in the WSN.

\section{RESULTS AND ANALYSIS}

Here, 2D and 3D WSNs are considered with less complex topology and energy-efficient routing protocols. Using Matlab, proposed ACO is simulated and compared with conventional ACO. In figure 5, total energy is considered with sensing period, which allows us to calculate the EE. Within the selected time, proposed and minimum energy can be obtained.

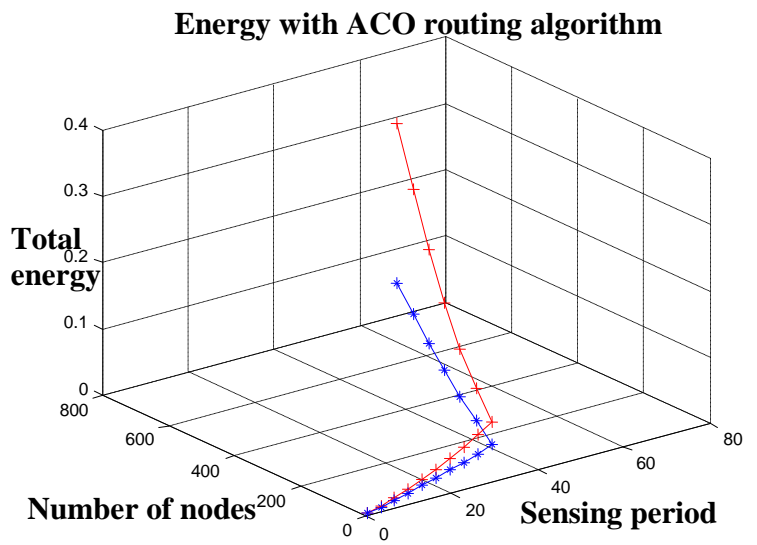

Fig 4: Comparisons of total energy

$\mathrm{EE}\left(E_{\eta}\right)$ can be defined as in (9) with minimum energy $E_{\text {min }}$ used in WSN and selected scheme $E_{\text {Sel }}$ involved with 
specific design and routing protocol.

$E_{\eta}=E_{\min } / E_{\text {sel }}$

Here, selected scheme is considered as a proposed method, which is simulated for measuring the used energy in transmitter and receiver of WSN. Following figures 5 and 6 are simulated using flowchart given in figure 2 .

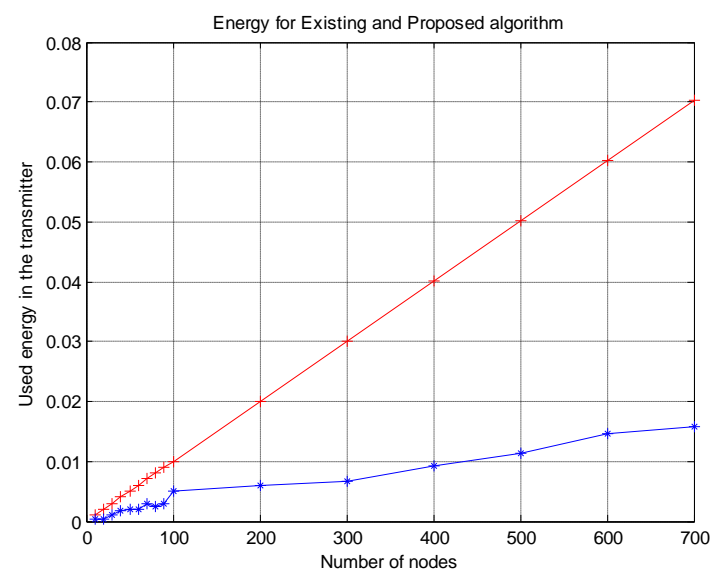

Fig 5: Comparisons of used energy for transmitter

As shown in figures 5 and 6, energy difference between the transmitter and receiver is very small because selected topology had less complexity. It means CT is very small because path selection using proposed algorithm is very efficient.

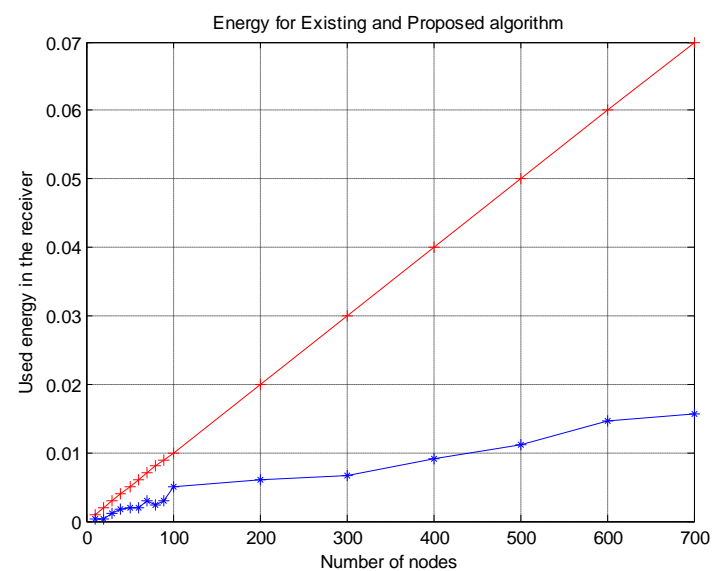

Fig 6: Comparisons of used energy for receiver

Using above simulations, EE can be calculated using (9) where minimum energy from existing scheme and selected energy from proposed scheme should be used. Both cases, fixed time and same topology must be considered.

\section{CONCLUSIONS}

The topology of existing WSN and three dimensional WSN have been studied for EE analysis. In this research, specific topology is used in energy efficient framework designed using with routers and routing protocols.

In this article, we analyzed the EE using existing and proposed routing protocol which is an enhanced version of AOC used in WSN. The proposed protocol based on specific algorithm (ECL-AOC) is designed successfully with energy concepts.

From the initial results, proposed research has achieved better energy performance in both transmitter and receiver nodes of the WSN. Using these improvements, EE can be obtained.

In future work, external environments, traffic (congestion) problems with different types of delays used during the processing and link establishments will be considered for other topologies and routing algorithms.

\section{REFERENCES}

[1] Muhamad, Wan Norsyafizan W., et al. "Maximizing Network Lifetime with Energy Efficient Routing Protocol for Wireless Sensor Networks." MEMS, NANO, and Smart Systems (ICMENS), 2009 Fifth International Conference on. IEEE, 2009.

[2] H. Van Luu, X. Tang, "An efficient algorithm for scheduling sensor data collection through multi-path routing structures" Journal of Network and Computer Applications, vol. 38 (2014) pp.150-162.

[3] W. Guo, W. Zhang, "A survey on intelligent routing protocols in wireless sensor networks "Journal of Network and Computer Applications vol. 38, 2014, pp. 185-201.

[4] Nallusamy, R., et al. "Energy efficient dynamic shortest path routing in wireless Ad hoc sensor networks using genetic algorithm." Wireless Communication and Sensor Computing, 2010. ICWCSC 2010. International Conference on. IEEE, 2010.

[5] Wang, Jin, et al. "An energy efficient and load balancing routing algorithm for wireless sensor networks," Computer Science and Information Systems ComSIS 8.4 (2011): 991-1007.

[6] Bhattacharya, S., \& Bandyapadhyay, S. (2011, April). A dynamic energy efficient multi hop routing technique using energy aware clustering in wireless sensor network. In Electronics Computer Technology (ICECT), 2011 3rd International Conference on (Vol. 5, pp. 199202). IEEE.

[7] Chang, B., \& Zhang, X., An energy-efficient routing algorithm for data gathering in wireless sensor networks. In Cross Strait Quad-Regional Radio Science and Wireless Technology Conference (CSQRWC), 2012 (pp. 137-141). IEEE.

[8] A. Okazaki and A. Frohlich, "Ant-based dynamic hop optimization protocol: A routing algorithm for mobile wireless sensor networks," in GLOBECOM Workshops (GC Wkshps), 2011 IEEE, Dec. 2011, pp. 1139 -1143.

[9] Chang and Ju, "An efficient cluster-based power saving scheme for wireless sensor networks", EURASIP Journal on Wireless Communications and Networking 2012.

[10] A. M. Zungeru, L. M. Ang, S. R. S. Prabaharan, and K. P. Seng, "Ant based routing protocol for visual sensors," in Proceedings of the Informatics Engineering and Information Science Communications in Computer and Information Science (ICIEIS '11), A. AbdManaf et al., Ed., pp. 250-264, 2011.

[11] M. Saleem, I.Ullah, and M. Farooq, "BeeSensor: a beeinspired, energy-efficient and scalable routing protocol 
for wireless sensor networks," Information Science, vol. 200, pp. 38-56, 2012.

[12] K. Zeng, K. Ren, W. Lou, and P. J. Moran, "Energy aware efficient geographic routing in lossy wireless sensor networks with environmental energy supply," Wirel. Netw., vol. 15, no. 1, pp. 39-51, 2009.

[13] L. Zhichu Lin and M. van der Schaar, "Autonomic and distributed joint routing and power control for delaysensitive applications in multi-hop wireless networks," IEEE Transactions on Wireless Communications, vol. 10, no. 1, pp. 102-113, 2011.

[14] Chang, Ruay-Shiung, and Chia-Jou Kuo. "An energy efficient routing mechanism for wireless sensor networks." Advanced Information Networking and Applications, 2006. AINA 2006. 20th International Conference on. Vol. 2. IEEE, 2006.

[15] Liu, Wenjun, and Jiguo Yu. "Energy efficient clustering and routing scheme for wireless sensor networks." Intelligent Computing and Intelligent Systems, 2009. ICIS 2009. IEEE International Conference on. Vol. 3. IEEE, 2009.
[16] F. Liu, C. Y. Tsui, and Y. J. Zhang, "Joint routing and sleep scheduling for lifetime maximization of wireless sensor networks," IEEE Transactions on Wireless Communications, vol. 9, no. 7, pp. 2258-2267, 2010.

[17] F. Weiwei, L. Feng, Y. Liu, Y. Fangnan, S. Lei, and S. Nishio, "Energy-efficient cooperative communication for data transmission in wireless sensor networks," IEEE Transactions on Consumer Electronics, vol. 56, no. 4, pp. 2185-2192, 2010.

[18] J. Abouei, J. D. Brown, K. N. Plataniotis, and S. Pasupathy, "On the energy efficiency of LT codes in proactive wireless sensor networks," IEEE Transactions on Signal Processing, vol. 59, no.3, pp. 1116-1127, 2011.

[19] Yu Wang, Chih-Wei Yi, Minsu Huang, and Fan Li, "Three Dimensional Greedy Routing in Large-Scale Random Wireless Sensor Networks", Ad Hoc Networks Journal (Elsevier), Volume: 11, Issue: 4, Pages: 13311344, June 2013. 